\title{
EDITORIAL
}

\section{Paradigm shifts in lung cancer as defined in the new IASLC/ATS/ERS lung adenocarcinoma classification}

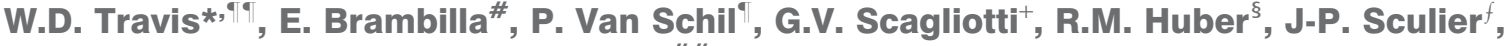 \\ J. Vansteenkiste** and A.G. Nicholson ${ }^{\# \#}$
}

$\mathbf{T}$ he new international multidisciplinary lung adenocarcinoma classification is sponsored by the European Respiratory Society (ERS), as well as the International Association for the Study of Lung Cancer (IASLC) and the American Thoracic Society (ATS) [1]. This classification is now published in the Journal of Thoracic Oncology, the official journal of the IASLC. Under the scientific oversight of the ATS and ERS, the classification project involved a systematic review to provide an evidence-based foundation with specific recommendations.

For decades, the field of lung cancer has been relatively static with few major advances. However, in the past 5-6 yrs this has changed to a rapidly evolving field. At the heart of this change is the impact of the molecular discovery that the activating mutations in the epidermal growth factor receptor (EGFR) are a marker for response and improved progression-free survival with tyrosine kinase inhibitor (TKI) therapy compared to conventional chemotherapy in advanced lung adenocarcinoma patients [2-5].

In this editorial, we summarise a series of major paradigm shifts that are outlined in this classification that will result in major changes in the approach to diagnosis of lung cancer compared to those outlined in previous World Health Organization (WHO) classifications. While this classification is primarily focused on lung adenocarcinoma, many of the recommendations will impact on how all histologic types of lung cancer are diagnosed in the future. This classification is divided into two major components: classification based on resection specimens (table 1) and on small biopsies and cytology (table 2).

\footnotetext{
${ }^{*}$ Dept of Pathology, Memorial Sloan Kettering Cancer Center, New York, NY, USA. ${ }^{*}$ CHU Albert Michallon, Dept d'Anatomie et Cytologie Pathologiques, Grenoble, France. "Dept of Thoracic and Vascular Surgery, Antwerp University Hospital, Antwerp. ${ }^{f}$ Dept of Medicine, Institut Jules Bordet, Brussels, ${ }^{*}$ Dept of Pulmonology, University Hospitals Leuven, Leuven, Belgium. ${ }^{+}$Thoracic Oncology, University of Turin, Department of Clinical and Biological Sciences, Turin, Italy. ${ }^{5}$ Division of Respiratory Medicine, University of Munich-Campus Innenstadt, Munich, Germany. ${ }^{\# \#}$ Dept of Pathology, Royal Brompton Hospital, London, UK. "The authors wrote this editorial on behalf of the core panel of the International Association for the Study of Lung Cancer (IASLC), American Thoracic Society (ATS) and European Respiratory Society (ERS) classification of lung adenocarcinoma; for a list of panel members, please refer to the Acknowledgements section.
}

CORRESPONDENCE: W.D. Travis, Dept of Pathology, Memorial Sloan Kettering Cancer Center, 1275 York Ave, New York, NY 10065, USA. E-mail: travisw@mskcc.org

\section{MULTIDISCIPLINARY APPROACH}

Historically, tumour classification has been primarily been performed by the WHO with panels primarily consisting of pathologists [6-9]. Due to the many major advances that have occurred at each level (pathology, molecular biology, radiology, respiratory medicine, oncology and surgery) this current classification project was established utilising an international multidisciplinary panel of pathologists, clinicians (oncologists/pulmonologists), molecular biologists, radiologists and surgeons [1].

One of the major lessons learned from this effort is the need for a multidisciplinary approach to diagnosis of lung adenocarcinoma. The need for direct interaction between clinicians, radiologists, pathologists, surgeons and molecular biologists has become more important than ever before. The new classification outlines the roles of each of these specialists and it emphasises the settings in which multidisciplinary interaction is needed. Each institution needs to have a plan for ongoing multidisciplinary interaction between specialists to discuss problem cases and to strategise the optimal approach to advanced lung cancer patients. The latter is needed so optimal small biopsy and/or cytology specimens are obtained and processed in pathology, not only for diagnosis but also for molecular studies. The different aspects of this process are summarised below.

\section{CLASSIFICATION IN SMALL BIOPSIES AND CYTOLOGY Rationale for need to further classify nonsmall cell lung cancer}

Since $70 \%$ of lung cancer patients present in advanced stages and are deemed unresectable, the diagnosis is by necessity established based on small biopsies and/or cytology. In the new IASLC/ATS/ERS classification, for the first time in the history of lung cancer classification, standardised criteria and terminology have been proposed that apply to pathologic diagnosis of lung cancer in small biopsies and cytology (table 2). Prior WHO classifications did not address this topic [1].

Historically there has been no clinical need to classify nonsmall cell lung cancer (NSCLC) further to distinguish squamous cell carcinoma from adenocarcinoma or other histologic types. However, since the 2004 WHO classification was published, three major advances have made histologic subclassification of lung cancer very important for therapeutic decisions in advanced lung cancer patients, particularly the distinction between adenocarcinoma and squamous cell carcinoma [6]. In addition to the finding that tumours with EGFR mutations are 
associated with a better outcome and response to TKIs as first line therapy in advanced lung adenocarcinoma patients [2-5], it was found that bevacizumab is contraindicated in patients with advanced squamous cell carcinoma due to increased risk of life-threatening haemorrhage [10], and pemetrexed is more efficacious in patients with adenocarcinoma or NSCLC, not otherwise specified (NSCLC-NOS) compared to those with squamous cell carcinoma, with intermediate activity in those tumours classified as NSCLC-NOS (not otherwise specified) [11-13].

Until now, EGFR mutation testing has been largely regarded as an investigational test, but now it is an official recommendation of three major societies involved with lung cancer.

Based on these observations the following recommendations were made.

\section{Recommendation to perform molecular testing for EGFR mutation}

In patients with advanced lung adenocarcinoma, we recommend testing for EGFR mutation (strong recommendation, moderate quality evidence).

Remarks: this is a strong recommendation because potential benefits clearly outweigh harms. This recommendation assumes that correct classification by EGFR mutation status is associated with important benefit based upon randomised phase 3 clinical trials of EGFR TKI therapy, which demonstrate a predictive benefit for response rate and progression-free survival, but not

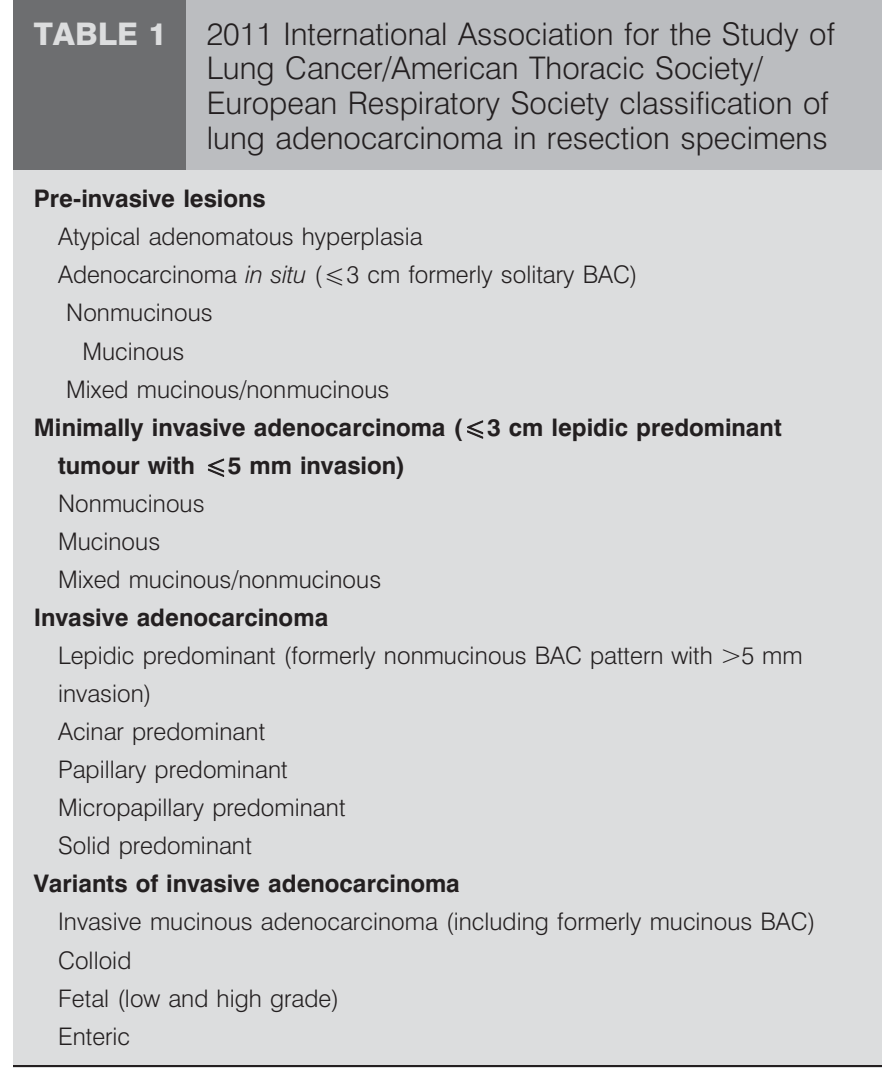

BAC: bronchioloalveolar carcinoma. overall survival, as well as subset analyses of multiple additional studies [1].

\section{Key pathology recommendations for small biopsies}

1) For small biopsies and cytology, we recommend that NSCLC be further classified into a more specific type, such as adenocarcinoma or squamous cell carcinoma, whenever possible (strong recommendation, moderate quality evidence).

2) We recommend that the term NSCLC-NOS be used as little as possible and we recommend it be applied only when a more specific diagnosis is not possible by morphology and/or special stains (strong recommendation, moderate quality evidence) [1].

\section{Pathology considerations for good practice:}

1) The term "non-squamous cell carcinoma" should not be used by pathologists in diagnostic reports. It is a categorisation used by clinicians to define groups of patients with several histologic types who can be treated in a similar manner; in small biopsies/cytology pathologists should classify NSCLC as adenocarcinoma, squamous cell carcinoma, NSCLC-NOS or other terms outlined in table 2 .

2) Tumours that are NSCLC-NOS by light microscopy with no morphologic features of adenocarcinoma or squamous differentiation that are further classified by special stains should be classified as "NSCLC, favour adenocarcinoma" or "NSCLC, favour squamous cell carcinoma". Cases that are not further classifiable by special stains remain as NSCLC-NOS (table 2).

3) Any workup of special stains to further classify cases that are NSCLC-NOS by light microscopy should be kept to a minimum to preserve as much material for molecular studies. It is suggested to use one adenocarcinoma marker and, if needed, one squamous marker or a mucin stain.

\section{New standardised diagnostic criteria and terminology for small biopsies and cytology}

In proposing the criteria and terminology for diagnosis of lung adenocarcinoma in small biopsies and cytology, the IASLC/ ATS/ERS committee had to make recommendations for terminology and criteria of other major histologic types of lung cancer because no such criteria had ever been recommended by the WHO (table 2).

In biopsies or cytology specimens, if squamous or adenocarcinoma differentiation is clearly present according to established morphologic criteria [6, 14, 15], tumours can be diagnosed with the established terms: "squamous cell carcinoma" and "adenocarcinoma".

According to the new classification, when a NSCLC does not show clear squamous or glandular differentiation by morphology in a small biopsy or cytology specimen, it is regarded as "nonsmall cell carcinoma not otherwise specified" ("NSCLCNOS") [1, 16]. If tissue is available, these tumours should be stained with a limited workup of immunohistochemical or mucin stains. If the tumour is positive for an adenocarcinoma marker or mucin, the diagnostic term is "NSCLC, favour adenocarcinoma". If the squamous cell carcinoma marker is positive and adenocarcinoma marker is negative, the diagnostic term is "NSCLC, favour squamous cell carcinoma". If the 
TABLE 2 Proposed International Association for the Study of Lung Cancer (IASLC)/American Thoracic Society (ATS)/European Respiratory Society (ERS) classification for small biopsies/cytology

\section{WHO Classification}

\author{
Adenocarcinoma \\ Mixed subtype \\ Acinar \\ Papillary
}

Solid

Bronchioloalveolar carcinoma (nonmucinous)

Bronchioloalveolar carcinoma (mucinous)

Fetal

Mucinous (colloid)

Signet ring

Clear cell

No 2004 WHO counterpart: most will be solid adenocarcinomas

Squamous cell carcinoma
Papillary
Clear cell
Small cell
Basaloid

No 2004 WHO counterpart

Small cell carcinoma

Small cell carcinoma
Large cell carcinoma
Large cell neuroendocrine carcinoma (LCNEC)
Large cell carcinoma with NE morphology (LCNEM)

\section{Small biopsy/cytology: 2011 IASLC/ATS/ERS}

Morphologic adenocarcinoma patterns clearly present:

Adenocarcinoma, describe identifiable patterns present (including micropapillary pattern not included in 2004 WHO classification)

Comment: if pure lepidic growth, mention an invasive component cannot be excluded in this small specimen

Adenocarcinoma with lepidic pattern (if pure, add note: an invasive component cannot be excluded)

Mucinous adenocarcinoma (describe patterns present)

Adenocarcinoma with fetal pattern

Adenocarcinoma with colloid pattern

Adenocarcinoma with (describe patterns present) and signet ring features

Adenocarcinoma with (describe patterns present) and clear cell features

Morphologic adenocarcinoma patterns not present (supported by special stains):

Nonsmall cell carcinoma, favour adenocarcinoma

Morphologic squamous cell patterns clearly present:

Squamous cell carcinoma

Morphologic squamous cell patterns not present (supported by stains):

Nonsmall cell carcinoma, favour squamous cell carcinoma

\section{Small cell carcinoma}

Nonsmall cell carcinoma, NOS

Nonsmall cell carcinoma with NE morphology (positive NE markers), possible LCNEC

Nonsmall cell carcinoma with NE morphology (negative NE markers); see comment

Comment: This is a non-small cell carcinoma where LCNEC is suspected, but stains failed to demonstrate NE differentiation

Adenosquamous carcinoma

Morphologic squamous cell and adenocarcinoma patterns present:

Nonsmall cell carcinoma, with squamous cell and adenocarcinoma patterns

Comment: this could represent adenosquamous carcinoma

No counterpart in 2004 WHO classification

Morphologic squamous cell or adenocarcinoma patterns not present but immunostains favour separate glandular and adenocarcinoma components

Nonsmall cell carcinoma, NOS (specify the results of the immunohistochemical stains and the interpretation)

Comment: this could represent adenosquamous carcinoma

Sarcomatoid carcinoma

Poorly differentiated nonsmall cell lung cancer with spindle and/or giant cell carcinoma (mention if adenocarcinoma or squamous carcinoma are present)

WHO: World Health Organization; NOS: not otherwise specified; NE: neuroendocrine. Reproduced from [1] with permission from the publisher. 
tumour cannot be classified after special stains are performed, it remains classified as NSCLC-NOS.

The main reasons for proposing the terms NSCLC, favour adenocarcinoma and NSCLC, favour squamous cell carcinoma are to enable: 1) pathologists to communicate to clinicians when the diagnosis was uncertain on morphology alone and that special stains were required to make the diagnosis; and 2) clinical trials to capture how the substantial percentage of NOS cases are being reclassified. In some of the major clinical trials, lung cancers have been classified as large cell carcinoma [11-13], but according to the 2004 WHO classification, that diagnosis cannot be established without a resection specimen due to the need for thorough histologic sampling to exclude the presence of any other differentiated component [6]. So these tumours probably represent NSCLC-NOS, lacking any clear morphologic differentiation on light microscopy in a small biopsy. In clinical trials this NOS category has been used in $20-40 \%$ of NSCLC $[11-13,17]$, and a recent epidemiological study suggests the frequency of this diagnosis has been increasing [17]. However, now these cases will be redistributed using immunohistochemistry or mucin stains into the categories of "NSCLC, favour adenocarcinoma", "NSCLC, favour squamous cell carcinoma" and a small percentage will remain unclassifiable as NSCLC-NOS. The term large cell carcinoma should not be used in the small biopsy or cytology setting, because this diagnosis requires evaluation of a resected specimen so the presence of other histologic components, such as adenocarcinoma or squamous cell carcinoma, can be excluded [6].

Therefore, future clinical trials need to document whether diagnoses are made based relying on special stains or by light microscopy alone. If no morphologic or immunohistochemical differentiation of adenocarcinoma or squamous cell carcinoma are identified, it should be classified as NSCLC-NOS.

Tumours that are candidates for EGFR mutation testing are those diagnosed as: 1) "adenocarcinoma", 2) "NSCLC, favor adenocarcinoma" or 3) "NSCLC-NOS" [1]. While the only molecular test recommended at this time is EGFR mutation testing in patients with these diagnoses in the setting of advanced lung cancer [1], it is recognised that in many institutions other molecular analyses will be performed, including KRAS mutation testing, although the presence of a KRAS mutation does not identify a therapeutic target at the present time, and testing for EML4-ALK translocation because these tumours are sensitive to specific agents such as crizotinib [18].

\section{Multidisciplinary strategy to manage small tissue and cytology samples}

Because of the need for molecular testing, each institution needs to develop a multidisciplinary strategy for obtaining and processing small biopsy and cytology specimens so that sufficient material is available not only for diagnosis but also for molecular studies. Small biopsies including formalin fixed, paraffin embedded tissues and/or cytologic samples including pleural fluids can be used for many molecular analyses, but the tissue needs to be managed carefully [19-21]. Preparation of cell blocks from cytology specimens is helpful for both immunohistochemical and molecular studies. This strategy needs to address the entire process beginning with the approach to obtaining biopsies or cytology specimens to how they are processed in pathology and then how they are delivered to the molecular laboratory and processed expeditiously so the results can be documented in a pathology report. As pathologists manage these specimens, they need to minimise the amount of tissue used for diagnostic workup and preserve as much material for molecular testing as possible [1, 16, 22].

\section{CLASSIFICATION IN RESECTION SPECIMENS}

For resection specimens, there are several important modifications of the $2004 \mathrm{WHO}$ classification. Several of these are addressed in the thoracic surgery article by VAN SCHIL et al. [23], so comments about these points will be brief. First, the concepts of adenocarcinoma in situ (AIS) and minimally invasive adenocarcinoma (MIA) are introduced for small solitary $(\leqslant 3 \mathrm{~cm})$ adenocarcinomas with either no invasion or only small foci of invasion measuring $\leqslant 0.5 \mathrm{~cm}$, respectively. AIS and MIA should define patients with either $100 \%$ or near $100 \% 5-y r$ disease free survival if completely resected. The term bronchioloalveolar carcinoma (BAC) is discontinued, because it falls into five different entities defined in this classification with disparate clinical and molecular properties and, as a result, it has caused great confusion. For overtly invasive adenocarcinomas the term mixed subtype is no longer used and tumours are classified according to their predominant subtype after using a method called comprehensive histologic subtyping to semiquantitatively estimate the percentages of the various subtypes present within the tumours. The term lepidic predominant adenocarcinoma is introduced for those nonmucinous tumours previously classified as mixed subtype in which the predominant subtype consists of the former nonmucinous BAC. Micropapillary adenocarcinoma is introduced as a histologic subtype, so the presence of a micropapillary pattern is estimated semiquantitavely and if it is the predominant pattern, it is classified as micropapillary predominant adenocarcinoma. Also, the tumours formerly classified as mucinous BAC are now reclassified into mucinous AIS or MIA or invasive mucinous adenocarcinoma, because a high percentage of these tumours have KRAS mutations, they often lack thyroid transcription factor expression, computed tomography (CT) frequently shows nodules of consolidation with air bronchograms and they are frequently multinodular and multilobar indistribution.

The new classification has several important implications for TNM staging. First, comprehensive histologic subtyping along with other histologic characteristics can be very helpful in determining if multiple pulmonary nodules are separate primaries or intrapulmonary metastases showing good correlation with molecular findings and clinical outcome [24-26]. Furthermore, it may be possible to improve TNM staging for lung adenocarcinomas that have a lepidic component by using the invasive size to predict tumour size rather than total size both by pathologic and CT assessment. There is already data in the pathology and CT literature that suggests this may be true [27-30]. If this turns out to be validated in future studies, in the next edition of the TNM it may be possible to use new terms for AIS and MIA, so Tis (adenocarcinoma) can be used for AIS and Tmi (adenocarcinoma) for MIA. These changes in TNM await the next revision but hopefully sufficient data will accumulate by that time to allow the TNM committee to address this issue. 


\section{STATEMENT OF INTEREST}

Statements of interest for G.V. Scagliotti and A.G. Nicholson can be found at www.erj.ersjournals.com/site/misc/statements.xhtml

\section{ACKNOWLEDGEMENTS}

The core panel of the International Association for the Study of Lung Cancer (IASLC), American Thoracic Society (ATS) and European Respiratory Society (ERS) classification of lung adenocarcinomab are as follows. M. Noguchi, Tsukuba, Japan; K. Geisinger, Winston-Salem, NC, USA; Y. Yatabe, Nagoya, Japan; D. Beer, Ann Arbor, MI, USA C.A. Powell, New York, NY, USA; G.J. Riely, New York, NY, USA; K. Garg, Aurora, CO, USA; J. Austin, New York, NY, USA; H. Asamura, Tokyo, Japan; V. Rusch, New York, NY, USA; F. Hirsch, Aurora, CO, USA; Y. Ishikawa, Tokyo, Japan; J. Jett, Denver, CO, USA; T. Mitsudomi, Nagoya, Japan; M. Sanchez-Cespedes, Barcelona, Spain; T. Takahashi, Nagoya City, Japan; M. Tsuboi, Tokyo, Japan; I. Wistuba, Houston, TX, USA; P-C. Yang, Taipei, Taiwan; D. Aberle, Los Angeles, CA, USA; C. Brambilla, Grenoble, France; D.B. Flieder, Philadelphia, PA, USA; W. Franklin, Aurora, CO, USA; A. Gazdar, Dallas, TX, USA; M.K. Gould, Los Angeles, CA, USA; P.S. Hasleton, Manchester, UK; D.W. Henderson, Adelaide, Australia; B. Johnson, Boston, MA, USA; D. Johnson, Dallas, TX, USA; K.M. Kerr, Aberdeen, UK; K. Kuriyama, Osaka, Japan; J. S. Lee, Seoul, Korea; V. Miller, New York, NY, USA; I. Petersen, Jena, Germany; V. Roggli, Durham, NC, USA; R. Rosell, Barcelona, Spain; N. Saijo, Osaka, Japan; E. Thunnissen, Amsterdam, the Netherlands; M. Tsao, Toronto, ON, Canada; D. Yankelewitz, New York NY, USA.

\section{REFERENCES}

1 Travis WD, Brambilla E, Noguchi M, et al. The New IASLC/ATS/ ERS International Multidisciplinary Lung Adenocarcinoma Classification. J Thoracic Oncol 2011; 6: 244-285.

2 Mok TS, Wu YL, Thongprasert S, et al. Gefitinib or carboplatinpaclitaxel in pulmonary adenocarcinoma. N Engl J Med 2009; 361: 947-957.

3 Mitsudomi T, Morita S, Yatabe Y, et al. Gefitinib versus cisplatin plus docetaxel in patients with non-small-cell lung cancer harbouring mutations of the epidermal growth factor receptor (WJTOG3405): an open label, randomised phase 3 trial. Lancet Oncol 2010; 11: 121-128.

4 Maemondo M, Inoue A, Kobayashi K, et al. Gefitinib or chemotherapy for non-small-cell lung cancer with mutated EGFR. N Engl J Med 2010; 362: 2380-2388.

5 Zhou C, Wu Y-L, Chen G, et al. Efficacy results from the randomized phase III OPTIMAL (CTONG 0802) study comparing first-line erlotinib versus carboplatin (CBDCA) plus gemcitabine (GEM) in Chinese advanced non-small cell lung cancer (NSCLC) patients (PTS) with EGFR activating mutations. Ann Oncol 2010; 21: LBA 13.

6 Travis WD, Brambilla E, Müller-Hermelink HK, et al. Pathology and Genetics: Tumours of the Lung, Pleura, Thymus and Heart. Lyon, IARC, 2004.

7 Travis WD, Colby TV, Corrin B, et al. Histological Typing of Lung and Pleural Tumors. Third Edn. Berlin, Springer, 1999.

8 World Health Organization. Histological Typing of Lung Tumours. First Edn. Geneva, World Health Organization, 1967.

9 World Health Organization. Histological Typing of Lung Tumours. Second Edn. Geneva, World Health Organization, 1981.

10 Johnson DH, Fehrenbacher L, Novotny WF, et al. Randomized phase II trial comparing bevacizumab plus carboplatin and paclitaxel with carboplatin and paclitaxel alone in previously untreated locally advanced or metastatic non-small-cell lung cancer. J Clin Oncol 2004; 22: 2184-2191.

11 Syrigos KN, Vansteenkiste J, Parikh $\mathrm{P}$, et al. Prognostic and predictive factors in a randomized phase III trial comparing cisplatin-pemetrexed versus cisplatin-gemcitabine in advanced non-small-cell lung cancer. Ann Oncol 2010; 21: 556-561.

12 Ciuleanu $\mathrm{T}$, Brodowicz $\mathrm{T}$, Zielinski $\mathrm{C}$, et al. Maintenance pemetrexed plus best supportive care versus placebo plus best supportive care for non-small-cell lung cancer: a randomised, double-blind, phase 3 study. Lancet 2009; 374: 1432-1420.

13 Scagliotti GV, Parikh P, von PJ, et al. Phase III study comparing cisplatin plus gemcitabine with cisplatin plus pemetrexed in chemotherapy-naive patients with advanced-stage non-small-cell lung cancer. J Clin Oncol 2008; 26: 3543-3551.

14 Foot NC. The identification of types of pulmonary cancer in cytologic smears. Am J Pathol 1952; 28: 963-983.

15 Johnston WW. Cytologic diagnosis of lung cancer. Principles and problems. Pathol Res Pract 1986; 181: 1-36.

16 Travis WD, Rekhtman N, Riley GJ, et al. Pathologic diagnosis of advanced lung cancer based on small biopsies and cytology: a paradigm shift. J Thorac Oncol 2010; 5: 411-414.

$17 \mathrm{Ou} \mathrm{SH}$, Zell JA. Carcinoma NOS is a common histologic diagnosis and is increasing in proportion among non-small cell lung cancer histologies. J Thorac Oncol 2009; 4: 1202-1211.

18 Kwak EL, Bang YJ, Camidge DR, et al. Anaplastic lymphoma kinase inhibition in non-small-cell lung cancer. N Engl J Med 2010 28, 363: 1693-1703.

19 Li AR, Chitale D, Riely GJ, et al. EGFR mutations in lung adenocarcinomas: clinical testing experience and relationship to EGFR gene copy number and immunohistochemical expression. J Mol Diagn 2008; 10: 242-248.

20 Lim EH, Zhang SL, Li JL, et al. Using whole genome amplification (WGA) of low-volume biopsies to assess the prognostic role of EGFR, KRAS, p53, and CMET mutations in advanced-stage nonsmall cell lung cancer (NSCLC). J Thorac Oncol 2009; 4: 12-21.

21 Solomon SB, Zakowski MF, Pao W, et al. Core needle lung biopsy specimens: adequacy for EGFR and KRAS mutational analysis. AJR Am J Roentgenol 2010; 194: 266-269.

22 Rossi G, Papotti M, Barbareschi M, et al. Morphology and a limited number of immunohistochemical markers may efficiently subtype non-small-cell lung cancer. J Clin Oncol 2009; 27: e141-e142.

23 Van Schil PE, Asamura H, Rusch V, et al. Surgical implications of the new IASLC/ATS/ERS lung adenocarcinoma classification. Eur Respir J 2011; submitted.

24 Girard N, Shen R, Guo T, et al. Comprehensive genomic analysis reveals clinically relevant molecular distinctions between thymic carcinomas and thymomas. Clin Cancer Res 2009; 15: 6790-6799.

25 Girard N, Deshpande C, Lau C, et al. Comprehensive histologic assessment helps to differentiate multiple lung primary nonsmall cell carcinomas from metastases. Am J Surg Pathol 2009; 33: 1752-1764.

26 Finley DJ, Yoshizawa A, Travis W, et al. Predictors of outcomes after surgical treatment of synchronous primary lung cancers. J Thorac Oncol 2010; 5: 197-205.

27 Yoshizawa A, Motoi N, Riely GJ, et al. Impact of proposed IASLC/ ATS/ERS classification of lung adenocarcinoma: prognostic subgroups and implications for further revision of staging based on the analysis of 514 stage I cases. Mod Pathol 2011; 24: 653-664.

28 Borczuk AC, Qian F, Kazeros A, et al. Invasive size is an independent predictor of survival in pulmonary adenocarcinoma. Am J Surg Pathol 2009; 33: 462-469.

29 Bhure UN, Lardinois D, Kalff V, et al. Accuracy of CT parameters for assessment of tumour size and aggressiveness in lung adenocarcinoma with bronchoalveolar elements. Br J Radiol 2010; 83: 841-849.

30 Lee HY, Han J, Lee KS, et al. Lung adenocarcinoma as a solitary pulmonary nodule: prognostic determinants of CT, PET, and histopathologic findings. Lung Cancer 2009; 66: 379-385. 